\title{
Canadian
} Science Publishing

Canadian Journal of Physics

Revue canadienne de physique

\section{Electron Bernstein Waves in a Collisionless Magnetoplasma with Cairns Distribution Function}

\begin{tabular}{|c|c|}
\hline Journal: & Canadian Journal of Physics \\
\hline Manuscript ID & cjp-2017-0044.R1 \\
\hline Manuscript Type: & Article \\
\hline Date Submitted by the Author: & 11-May-2017 \\
\hline Complete List of Authors: & $\begin{array}{l}\text { Malik, Mohammad ; Quaid-i-Azam University, Physics } \\
\text { Masood, Waqas; COMSATS institute of Information technology, Physics } \\
\text { Rehman, Aman-ur; PIEAS, } \\
\text { Mirza, Arshad; Quaid-i-Azam University } \\
\text { Qamar, Anisa; University of Peshawar , Physics }\end{array}$ \\
\hline Keyword: & $\begin{array}{l}\text { Electron Bernstein Waves, Collisionless Magnetoplasma, Cairns Distribution } \\
\text { Function, Electrostatic Electrons, Non-thermal Parameter }\end{array}$ \\
\hline $\begin{array}{r}\text { Is the invited manuscript for } \\
\text { consideration in a Special } \\
\text { Issue? : }\end{array}$ & Ursula Franklin commemorative Festschrift \\
\hline
\end{tabular}




\title{
Electron Bernstein waves in a collisionless magnetoplasma with Cairns distribution function
}

\author{
M. Usman Malik ${ }^{1}$, W. Masood ${ }^{2,3}$, Aman-ur-Rehman $^{4}$, Arshad M. Mirza ${ }^{1}$ and Anisa Qamar ${ }^{5}$ \\ 1) Theoretical Plasma Physics Group, Quaid-i-Azam \\ University, Islamabad 44000, Pakistan. \\ ${ }^{2)}$ COMSATS Institute of Information Technology, \\ Park Road, Chak Shahzad, Islamabad 44000, Pakistan. \\ ${ }^{3)}$ National Center for Physics, Islamabad 44000, \\ Pakistan. \\ 4) Pakistan Institute of Engineering and Applied sciences, \\ P. O. Nilore, Islamabad, Pakistan. \\ ${ }^{5)}$ Department of Physics University of Peshawar, Peshawar 25000, \\ Pakistan.
}

May 11, 2017

\begin{abstract}
In this paper, we have investigated the electrostatic electron Bernstein waves in a collisionless magnetized plasma using the Cairns distribution function. In this regard, we have derived a generalized dielectric constant for the Bernstein waves and derived the modified dispersion relation in the presence of Cairns distribution function. We have found that the dispersion curves for the electron Bernstein waves using the Cairns distribution
\end{abstract}


function show a very significant deviation from the Maxwellian results. It has been found that the behavior of the Bernstein waves across the entire band between the adjacent harmonics show a departure from the Maxwellian result for the different values of the non-thermality parameter for the Cairns distribution function.

\section{Introduction}

The satellite missions in astrophysical and space plasmas have regularly observed particle distribution functions that are quasi-Maxwellian up to the mean thermal velocities and possess non-Maxwellian suprathermal tails at high velocities or energies [1], [2]. These so-called nonthermal plasmas have been ubiquitously found in the magnetospheres of Earth, Mercury, Saturn, and Uranus, and in the solar wind [3], [4]. The observed distributions of charged particles have been well fitted with a generalized Lorentzian distribution, since it fits both the thermal as well as the suprathermal parts of the observed energy velocity spectra [1], [3], [5], [2], [6], [7]. The spectral index $\kappa$ quantifies the slope of the energy spectrum of the suprathermal particles forming the tail of the velocity distribution function; the smaller the value of $\kappa$, the more suprathermal particles in the distribution function tail and the harder the energy spectrum. kappa distributions approach the Maxwellian as $\kappa->\infty$.

The other commonly employed distribution to study the distributions far removed from Maxwellian distribution is Cairns distribution. Cairns distribution was successfully used to explain the rarefactive ion-acoustic solitary waves observed by the Freja satellite. Cairns et. al. [8] showed that the presence of a non-Maxwellian distribution of electrons could transfigure the nature of nonlinear density excitations to allow for the existence of structures observed by the Freja and Viking satellites [9], [10]. Various studies have shown that the non-Maxwellian distributions significantly modify the linear and nonlinear propagation of waves in plasmas[11], [12], [13].

Electron Bernstein waves were first derived by Bernstein in 1958 for a hot 
collisionless magnetoplasma employing the kinetic theory of plasmas [14]. Their presence in the laboratory plasmas was reported by Crawford and Leuterer in 1965 and 1969 respectively [15], [16]. The existence of the electrostatic waves near the harmonics of the gyro-frequency was a totally new effect and had no counterpart in the fluid theory. It was anticipated that there might be some cyclotron damping for the hot plasma in the direction perpendicular to the ambient magnetic field but none was found. It was later realized that this happens because for zero parallel propagation vector, the resonance velocity turns out to be infinite and hence no particles resonate with the waves. However, the inclusion of a non-zero parallel propagation vector showed the reappearance of the cyclotron damping [14].

Electron Bernstein waves are a useful diagnostic tool for spherical tokamaks and can be used for measurement of the local electron temperature if one can detect their intensities and identify their birth positions [17]. In space plasmas, these waves have also been routinely observed by spacecrafts, emitted from the magnetized plasma of Jupiter moon Io [18]. Mace [19] studied the electron Bernstein waves for isotropic kappa distribution, and derived the dispersion relation using Gordeyev-type integral. Later, he extended the work for explaining the banded emission in planetary magnetospheres. Meyer-Vernet et al. [20] showed that the weakly banded emission from the Jupiter magnetosphere between consecutive gyroharmonics frequency is quasithermal noise in Bernstein waves. Deeba et. al. [21] investigated the electron Bernstein waves using the kappa and r,q distribution and found that the both the distributions show a deviation from the Maxwellian results.

In the present investigation, by employing the kinetic theory of plasmas, we study the electron Bernstein waves in a hot collisionless magnetoplasma using the Cairns distribution function. The manuscript is organized in the following fashion. In Sec 2, we have presented a mathematical model for Bernstein waves using the linearized Vlasov model. In Section 3, we have derived the generalized dielectric constant for a hot, magnetized, collisionless plasma using the Cairns distribution function. In Section 4, we have presented the results and discussion 
and highlighted the differences of our results from the Maxwellian case. Finally, in Section 5, we have recapitulated the main results of our investigation.

\section{Kinetic treatment of electron Bernstein waves}

Consider a uniform, collisionless, hot plasma embedded in a constant magnetic field such that $\mathbf{B}_{o}=B_{o} \hat{z}$. We use standard Vlasov model to study the electrostatic Bernstein waves. It is pertinent to mention here that electron Bernstein waves are purely kinetic and have no counterpart in fluid theory. The ion contribution has been ignored here owing to its much larger mass by comparison with electrons. The wave propagation vector $\mathbf{k}$ is confined to the $x z$-plane so that $k=\left(k_{\perp}, 0, k_{\|}\right)$and $v=\left(v_{\perp} \cos \psi, v_{\perp} \sin \psi, v_{\|}\right)$. Vlasov equation can then be written as

$$
\partial_{t} f+\mathbf{v} \cdot \nabla_{x} f+\mathbf{a} \cdot \nabla_{v} f=0
$$

where $\mathbf{a}=-e / m_{e}[(\mathbf{E}+\mathbf{v} \times \mathbf{B})]$. For linearization we split the distribution function into the unperturbed and perturbed parts i.e. $f=f_{o}+f_{1}$. Similarly $E=E_{1}$ and $B_{1}=0$ since we are dealing with electrostatic waves. After linearization and collecting the first order terms we have

$$
\partial_{t} f_{1}+\mathbf{v} \cdot \boldsymbol{\nabla}_{x} f_{1}-\frac{e}{m_{e}}\left(\mathbf{v} \times \mathbf{B}_{0}\right) \cdot \boldsymbol{\nabla}_{v} f_{1}=\frac{e}{m_{e}}\left(\mathbf{E}_{1}\right) \cdot \boldsymbol{\nabla}_{v} f_{e 0} .
$$

For electrostatic case, we substitute $E_{1}=-\nabla \phi_{1}$ in equation (1) and obtain

$$
-i \omega f_{1}+i \mathbf{k} \cdot \mathbf{v} f_{1}-\frac{e}{m_{e}}\left(\mathbf{v} \times \mathbf{B}_{0}\right) \cdot \nabla_{v} f_{1}=-\frac{e}{m_{e}} \mathbf{k} \cdot \nabla_{v} f_{e 0} \phi_{1} .
$$

Rearranging the above equation yields

$$
\frac{\partial f_{1}}{\partial \psi}-\frac{(s+i \mathbf{k} \cdot \mathbf{v}) f_{1}}{\omega_{c e}}=\frac{i e \mathbf{k} \cdot \nabla_{v} f_{e 0} \phi_{1}}{m_{e} \omega_{c e}}
$$

which is the linear differential equation of first order for $f_{1}$, where, $s=-i \omega$ and $\omega_{c e}=\left|e B_{0} / m_{e}\right|$. The solution of Eq.(3) can be written as

$f_{1}=-\frac{i e \phi_{1}}{m_{e}} \sum_{p, q} J_{p}\left(\frac{k_{\perp} v_{\perp}}{\omega_{c e}}\right) J_{q}\left(\frac{k_{\perp} v_{\perp}}{\omega_{c e}}\right) e^{i(p-q) \psi}\left(\frac{\left(\frac{p \omega_{c e}}{v_{\perp}}\right) \frac{\partial f_{e 0}}{\partial v_{\perp}}+k_{\|} \frac{\partial f_{e 0}}{\partial v_{\|}}}{s+i k_{\|} v_{\|}+i p \omega_{c e}}\right)$. 
The Gauss's law is

$$
\nabla \cdot E_{1}=-4 \pi e n_{e 0} \int f_{1} d \mathbf{v}
$$

and upon substituting $E_{1}=-\nabla \phi_{1}$, we get

$$
k^{2} \phi_{1}=-4 \pi e n_{e 0} \int f_{1} d \mathbf{v}
$$

Inserting the value of the perturbed distribution function from Eq. (4) in Eq. (6) yields

$$
\left.\begin{array}{rl}
1-\sum_{p, q} \frac{i \omega_{p e}^{2}}{k^{2}} \int & J_{p}\left(\frac{k_{\perp} v_{\perp}}{\omega_{c e}}\right) J_{q}\left(\frac{k_{\perp} v_{\perp}}{\omega_{c e}}\right) \exp [i(p-q) \psi] \\
& \times\left(\frac{\left(\frac{p \omega_{c e}}{v_{\perp}}\right) \frac{\partial f_{e 0}}{\partial v_{\perp}}+k_{\|} \frac{\partial f_{e 0}}{\partial v_{\|}}}{s+i k_{\|} v_{\|}+i p \omega_{c e}}\right) d \overline{\mathbf{v}}
\end{array}\right) k^{2} \phi_{1}=0
$$

As $\int_{0}^{2 \pi} \exp [i(p-q) \psi] d \psi$ exists only if $p=q$, therefore the generalized dielectric relation for the electrostatic Bernstein waves will be [14]

$$
\epsilon(k, \omega)=0=1-\sum_{p=-\infty}^{\infty} \frac{i \omega_{p e}^{2}}{k^{2}} \int J_{p}^{2}\left(\frac{k_{\perp} v_{\perp}}{\omega_{c e}}\right)\left(\frac{\left(\frac{p \omega_{c e}}{v_{\perp}}\right) \frac{\partial f_{e 0}}{\partial v_{\perp}}+k_{\|} \frac{\partial f_{e 0}}{\partial v_{\|}}}{s+i k_{\|} v_{\|}+i p \omega_{c e}}\right) d \overline{\mathbf{v}}
$$

\section{Wave Propagation Perpendicular To B}

In order to consider only the perpendicular part of the wave from the magnetic field, we put $k_{\|}=0, \vec{k}=k_{\perp} \hat{x}, s=-i \omega$ in Eq.(8) and obtain

$$
\epsilon(k, \omega)=0=1+\sum_{p=-\infty}^{\infty} \frac{\omega_{p e}^{2}}{k^{2}} \int J_{p}^{2}\left(\frac{k_{\perp} v_{\perp}}{\omega_{c e}}\right)\left(\frac{\left(\frac{p \omega_{c e}}{v_{\perp}}\right) \frac{\partial f_{e 0}}{\partial v_{\perp}}}{\omega-p \omega_{c e}}\right) d \overline{\mathbf{v}}
$$

where $f_{o}$ is the equilibrium distribution function which is Cairns distribution for our case and is given by

$$
f_{e 0}=\frac{1}{(2 \pi)^{3 / 2}(3 \Gamma+1) v_{t h}^{3}}\left(1+\frac{\Gamma v_{e}^{4}}{v_{t h}^{4}}\right) \exp \left[-\frac{v_{e}^{2}}{2 v_{t h}^{2}}\right]
$$


where $\Gamma$ is non thermal parameter representing the percentage of the nonthermal population of electrons and $v_{t h}^{2}=K T / m_{e}$. Using the identity $J_{-p}\left(\frac{k_{\perp} v_{\perp}}{\omega_{c e}}\right)=$ $(-1)^{p} J_{p}\left(\frac{k_{\perp} v_{\perp}}{\omega_{c e}}\right)$, Eq. (9) becomes

$$
\epsilon(k, \omega)=1+\sum_{p=1}^{\infty} \frac{\omega_{p e}^{2}}{k^{2}} \int J_{p}^{2}\left(\frac{k_{\perp} v_{\perp}}{\omega_{c e}}\right)\left(\frac{1}{v_{\perp}}\right) \frac{\partial f_{e 0}}{\partial v_{\perp}}\left(\frac{2 p^{2} \omega_{c e}^{2}}{\omega^{2}-p^{2} \omega_{c e}^{2}}\right) d \overline{\mathbf{v}}
$$

\section{Bernstein Waves For Cairns Distribution}

Taking the derivative of the distribution function ( i.e. Eq. (10)) w.r.t. the perpendicular velocity and substituting it in Eq. (11) yields

$$
\begin{aligned}
& \epsilon(k, \omega)=0=1+\sum_{p=1}^{\infty} \frac{\omega_{p e}^{2}}{k^{2}} \int J_{p}^{2}\left(\frac{k_{\perp} v_{\perp}}{\omega_{c e}}\right) \frac{1}{(2 \pi)^{3 / 2}(3 \Gamma+1) v_{t h}^{3}}\left(\frac{2 p^{2} \omega_{c e}^{2}}{\omega^{2}-p^{2} \omega_{c e}^{2}}\right) \times \\
& \frac{1}{v_{\perp}}\left(v_{\perp}\left(\Gamma \frac{4 v_{\|}^{2}}{v_{t h}^{4}}-\frac{1}{v_{t h}^{2}}-\Gamma \frac{v_{\|}^{4}}{v_{t h}^{6}}\right)+v_{\perp}^{3}\left(\Gamma \frac{4}{v_{t h}^{4}}-\Gamma \frac{2 v_{\|}^{2}}{v_{t h}^{6}}\right)-\Gamma \frac{v_{\perp}^{5}}{v_{t h}^{6}}\right) \exp \left(-\frac{\left(v_{\perp}^{2}+v_{\|}^{2}\right)}{2 v_{t h}^{2}}\right) d \overline{\mathbf{v}}
\end{aligned}
$$

We first consider the perpendicular integral from above equation i.e.

$$
I_{1}=\int_{0}^{\infty} J_{p}^{2}\left(\frac{k_{\perp} v_{\perp}}{\omega_{c e}}\right)\left(v_{\perp}\left(\Gamma \frac{4 v_{\|}^{2}}{v_{t h}^{4}}-\frac{1}{v_{t h}^{2}}-\Gamma \frac{v_{\|}^{4}}{v_{t h}^{6}}\right)+v_{\perp}^{3}\left(\Gamma \frac{4}{v_{t h}^{4}}-\Gamma \frac{2 v_{\|}^{2}}{v_{t h}^{6}}\right)-\Gamma \frac{v_{\perp}^{5}}{v_{t h}^{6}}\right) \exp \left(-\frac{v_{\perp}^{2}}{2 v_{t h}^{2}}\right) d v_{\perp} .
$$

The solution of the above integral is

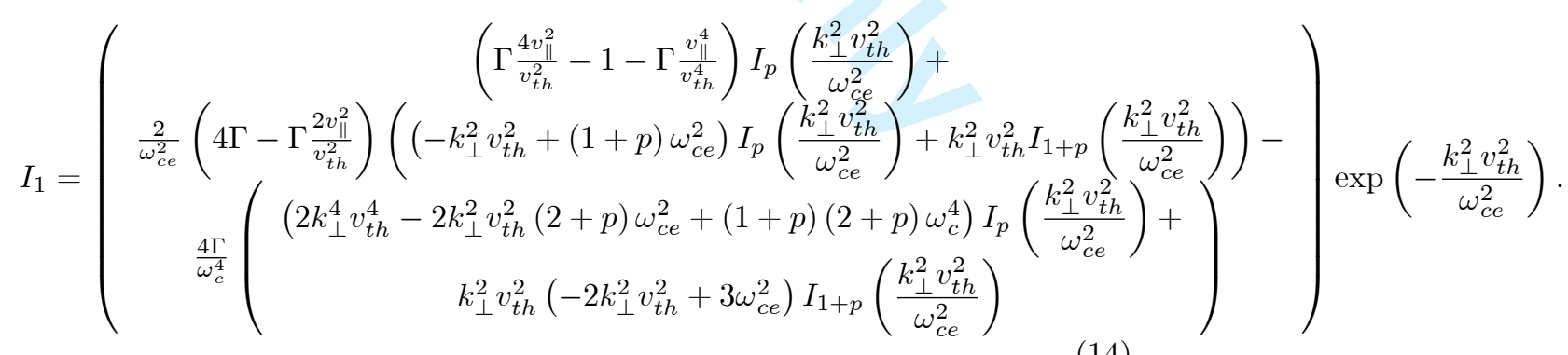


From Eq. (12), the parallel integral reads as

$$
\begin{aligned}
& I_{2}=\exp \left(-\frac{k_{\perp}^{2} v_{t h}^{2}}{\omega_{c e}^{2}}\right) \int_{-\infty}^{\infty} d v_{\|} \exp \left(-\frac{v_{\|}^{2}}{v_{t h}^{2}}\right) \times \\
& \left(\begin{array}{c}
\left(\Gamma \frac{4 v_{\|}^{2}}{v_{t h}^{2}}-1-\Gamma \frac{v_{\|}^{4}}{v_{t h}^{4}}\right) I_{p}\left(\frac{k_{\perp}^{2} v_{t h}^{2}}{\omega_{c e}^{2}}\right)+ \\
\frac{2}{\omega_{c e}^{2}}\left(4 \Gamma-\Gamma \frac{2 v_{\|}^{2}}{v_{t h}^{2}}\right)\left(\begin{array}{c}
\left(-k_{\perp}^{2} v_{t h}^{2}+(1+p) \omega_{c e}^{2}\right) I_{p}\left(\frac{k_{\perp}^{2} v_{t h}^{2}}{\omega_{c e}^{2}}\right)+ \\
k_{\perp}^{2} v_{t h}^{2} I_{1+p}\left(\frac{k_{\perp}^{2} v_{t h}^{2}}{\omega_{c e}^{2}}\right)
\end{array}\right)- \\
\frac{4 \Gamma}{\omega_{c}^{4}}\left(\begin{array}{c}
\left(2 k_{\perp}^{4} v_{t h}^{4}-2 k_{\perp}^{2} v_{t h}^{2}(2+p) \omega_{c e}^{2}+(1+p)(2+p) \omega_{c}^{4}\right) I_{p}\left(\frac{k_{\perp}^{2} v_{t h}^{2}}{\omega_{c e}^{2}}\right)+ \\
k_{\perp}^{2} v_{t h}^{2}\left(-2 k_{\perp}^{2} v_{t h}^{2}+3 \omega_{c e}^{2}\right) I_{1+p}\left(\frac{k_{\perp}^{2} v_{t h}^{2}}{\omega_{c e}^{2}}\right)
\end{array}\right)
\end{array}\right) \\
& =\sqrt{2 \pi} v_{t h}\left(\begin{array}{c}
-8 \Gamma \frac{k_{\perp}^{4} v_{t h}^{4}}{\omega_{c e}^{4}}+4 \Gamma \frac{k_{\perp}^{2} v_{t h}^{2}}{\omega_{c e}^{2}}(3+2 p)- \\
4 \Gamma(1+p)^{2}-1+\Gamma \\
8 \Gamma\left(\frac{k_{\perp}^{2} v_{t h}^{2}}{\omega_{c e}^{2}}\left(\frac{k_{\perp}^{2} v_{t h}^{2}}{\omega_{c e}^{2}}-1\right) I_{p}\left(\frac{k_{\perp}^{2} v_{t h}^{2}}{\omega_{c e}^{2}}\right)+\left(\frac{k_{\perp}^{2} v_{t h}^{2}}{\omega_{c e}^{2}}\right)\right)
\end{array}\right) \exp \left(-\frac{k_{\perp}^{2} v_{t h}^{2}}{\omega_{c e}^{2}}\right) .
\end{aligned}
$$

Inserting the results of perpendicular and parallel integrations in Eq. (12), we get

$$
\epsilon(k, \omega)=1-\sum_{p=1}^{\infty} \frac{2 \omega_{p e}^{2}}{\Theta^{2} \omega_{c e}^{2}} \frac{\Lambda_{p}(\Theta)}{\left(\frac{\omega}{p \omega_{c e}}\right)^{2}-1},
$$

where $\Theta=k_{\perp} v_{t h} / \omega_{c e}, I_{p}(b)$ is the modified Bessel function, $\omega_{p e}$ is the electron plasma frequency, $\omega_{c e}$ is the electron cyclotron frequency and the function $\Lambda_{p}(\Theta)$ contains all the modifications due to the presence of Cairn's distribution instead of Maxwellian distribution and is given by

$$
\Lambda_{p}(\Theta)=e^{-\Theta^{2}} \varpi
$$

where

$\varpi=\frac{\left\{\left(8 \Gamma \Theta^{4}-4 \Gamma \Theta^{2}(3+2 p)+4 \Gamma(1+p)^{2}+1-\Gamma\right)\right\} I_{p}\left(\Theta^{2}\right)+8 \Gamma\left[\Theta^{2}\left(1-\Theta^{2}\right) I_{1+p}\left(\Theta^{2}\right)\right]}{(3 \Gamma+1)}$ 
Putting $\Gamma=0$ gives us the dielectric function for the Maxwell-Boltzmann case (i.e. by putting $\varpi=I_{p}\left(\Theta^{2}\right)$ ). It can be seen that it turns out to be the same as given by Gurnett and Bhattacharjee [14].

\section{Results and Discussion}

In this paper, we have adopted the procedure used by Gurnett and Bhattacharjee [14] to derive the dispersion relation for the electron Bernstein waves using the Cairns distribution function. Equation (18) shows that the function $\Lambda_{p}$ gets significantly modified owing to the presence of nonthermality parameter $\Gamma$. It is pertinent to mention here that the function $\Lambda_{p}$ reduces to the function given

by Gurnett and Bhattacharjee [14] in the limit $\Gamma$ goes to zero. Plots of $\Lambda_{p}$ as function of $\Theta$ are shown in Figure (1) for the first three harmonics i.e. $n=1,2$ and 3. As can be seen, the $\Lambda_{p}$ functions all have a single maximum that decreases and shifts to large $\Theta$ values as $n$ increases. For $n=1$, the peak values are $\Theta=0.84,0.75$ and 0.72 for the corresponding values of $\Gamma=0.1,0.2$ and 0.3 respectively. Note that the peak values for $n=1$ corresponding to different values of nonthermality parameter show a departure from the Maxwellian case. This implies that the change in the shape of the distribution function significantly modifies the propagation charcteristics of Bernstein waves.

The general nature of the roots of the dispersion relation can be ascertained by plotting $\epsilon(k, \omega)$ as a function of $\omega$ for a specific value of $\Theta=k_{\perp} v_{t h} / \omega_{c e}$ and then searching for the points that yield $\epsilon(k, \omega)=0$. Notice that the thermal cyclotron radius $v_{t h} / \omega_{c e}$ is a basic scale factor in the perpendicular wavelength. Note that the non-Maxwellian distribution changes the temperature (since the width of the distribution function defines temperature) and hence the basic scale factor changes which is responsible for the observed departures from the Maxwellian behavior. Note also that as $k_{\perp} v_{t h} / \omega_{c e} \rightarrow 0$, the dispersion relation $\epsilon(k, \omega)$ approaches the cold plasma equation. A representative plot of $\epsilon(k, \omega)$ is shown in Figure $(2)$. Since $\epsilon(k, \omega)$ has a single-order pole at each of the gyro-harmonics, the roots of the dispersion relation consist of infinite number 
of frequencies, $\omega_{1}, \omega_{2}, \ldots$, with one root between each of the adjacent cyclotron harmonics. It can be seen that the change in the values of the nonthermality parameter enhances the value of $\epsilon(k, \omega)$, however, the propagation range of the electron Bernstein waves within the adjacent cyclotron harmonics decreases.

By solving the dispersion relation, $\epsilon(k, \omega)=0$, as a function of $\Theta$, the frequency of each mode, $\omega_{n}$, can be determined as a function of $\Theta$. This procedure must be done numerically. A typical plot of $\omega_{n}$ as a function of $\Theta$ is shown in Figure (3). For the parameter used, $\omega_{p e}=2.5 \omega_{c e}$, the ratio of the plasma frequency to the cyclotron frequency is such that the upper hybrid frequency, $\omega_{U H}$, is located between the second and third electron cyclotron frequency. However, it can be seen that unlike the Maxwellian case shown by the solid line, the values of the frequencies above the upper hybrid frequency are not restricted to a narrow frequency range, a radical departure from the Maxwellian case. It is instructive to mention here that no cyclotron damping occurs for the exact perpendicular propagation of electron Bernstein waves as that would imply an infinite resonant velocity with which no wave can resonate. However, for a non-zero parallel propagation vector, the damping reappears.

\section{Conclusions}

Employing the linear Vlasov kinetic theory, we have explored here the dispersion characteristics of the electrostatic electron Bernstein waves in a collisionless magnetized plasma using the Cairns distribution function. We have found that the Cairns distribution significantly modifies the dispersion relation of electron Bernstein waves. We have closely followed the procedure adopted by Gurnett and Bhattacharjee to derive the electron Bernstein waves where they were derived using the Maxwellian distribution. It has been found that the function used to plot the dispersion relation by Gurnett and Bhattacharjee [14] gets modified in the presence of Cairns distribution function. It has been shown that the peak values obtained by Gurnett and Bhattacharjee for the Maxwellian case undergo a change by the presence of nonthermality parameter of the Cairns 
distribution. It has been shown that this change leads to a modified behavior of electron Bernstein waves across the entire band between the adjacent harmonics.

\section{References}

[1] S. M. Krimigis, J. F. Carbary, E. P. Keath, T. P. Armstrong, L. J. Lanzerotti, and G. Gloeckler, J. Geophys. Res., 88, 8871 (1983).

[2] M. Maksimovic, V. Pierrard, and J. F. Lemaire, Astron. Astrophys., 324, 725 (1997).

[3] Christon, S. P., D. G. Mitchell, D. J. Williams, L. A. Frank, C. Y. Huang, and T. E. Eastman, J. Geophys. Res., 93, 2562 (1988).

[4] Pierrard, V., H. Lamy, and J. F. Lemaire, J. Geophys. Res., 109, A02118 (2004).

[5] Pierrard, V. and J. Lemaire, J. Geophys. Res., 101, 7923 (1996).

[6] A. F. Vinas, R. L. Mace, and R. F. Benson, J. Geophys. Res.,110, A06202 (2005).

[7] T. K. Baluku, M. A. Hellberg, and R. L. Mace, J. Geophys. Res.,116, A04227 (2011).

[8] R. A. Cairns, A. A. Mamun, R. Bingham, R. Boström, R. O. Dendy, C. M.C. Nairn, and P. K. Shukla, Geophys. Res. Lett., 22, 2709 (1995).

[9] R. Bostrom, IEEE Trans. Plasma Sci., 20, 756 (1992).

[10] P. O. Dovner, A. I. Eriksson, R. Bostrom, and B. Holback, Geophys. Res. Lett., 21, 1827 (1994).

[11] A. Saha, P. Chatterjee and C. S. Wang, Braz J. Phys 45, 325 (2015).

[12] A. Saha, N. Pal, T. Saha, M. K. Ghorui, P. Chatterjee, J. Theor Appl Phys 10, $271(2016)$. 
[13] R. Ali, A. Saha and P. Chatterjee, Indian J. Phys., DOI 10.1007/s12648017-0957-7 (2017).

[14] D. A. Gurnett and Amitava Bhattacharjee, Introduction to Plasma Physics with Space and Laboratory applications, Cambridge University Press, 2005).

[15] F. Crawford, J. Res. Natl. Bur. Stand. 69, 789 (1965).

[16] F. Leuterer, Plasma Phys. 11, 615 (1969).

[17] P. C. Efthimion, J. C. Hosea, R. Kaita, R. Majeski, and G. Taylore, Rev. Sci. Instrum. 70, 1018 (1999).

[18] M. Moncuquet, N. Meyer Vernet, and S. Hoang, J. Geophys. Res. 100, 21697 (1995).

[19] R. L. Mace, Phys. Plasmas 10, 2181 (2003).

[20] N. Meyer-Vernet, S. Hoang, and M. Moncuquet, J. Geophys. Res. 98, 21163 (1993).

[21] F. Deeba, Zahoor Ahmad, and G. Murtaza, Phys. Plasmas. 17, 102114 (2010). 


\section{Figure Captions}

Fig. 1: (Color Online) Plot of function $\Lambda_{p}(\Theta)$ versus $\Theta$ for $n=1,2$ and 3 for differernt values of non thermal parameter. Solid lines represent for $n=1$, dotdashed lines represent for $n=2$ and dashed lines represent for $n=3$. (Blue color is for $\Gamma=0$ (Maxwellian) Brown is for $\Gamma=0.1$, Black is for $\Gamma=0.2$ and Green color is for $\Gamma=0.3$.).

Fig. 2: (Color Online) Plot of Dielectric Constant $(\epsilon(k, \omega))$ versus normalized frequency $\omega / \omega_{c e}$ for different values of nonthermal parameter, Blue solid line is for $\Gamma=0$ (Maxwellian), Black dotted line is for $\Gamma=0.1$, Brown dotdashed line is for $\Gamma=0.2$ and Green dashed line is for $\Gamma=0.3$.

Fig. 3: (Color Online) Plots of $\omega / \omega_{c e}$ versus $\Theta$ for differernt values of nonthermal parameter. Blue solid line is for $\Gamma=0$ (Maxwellian), Black dotted line is for $\Gamma=0.1$, Brown dotdashed line is for $\Gamma=0.2$ and Green dashed line is for $\Gamma=0.3$. 


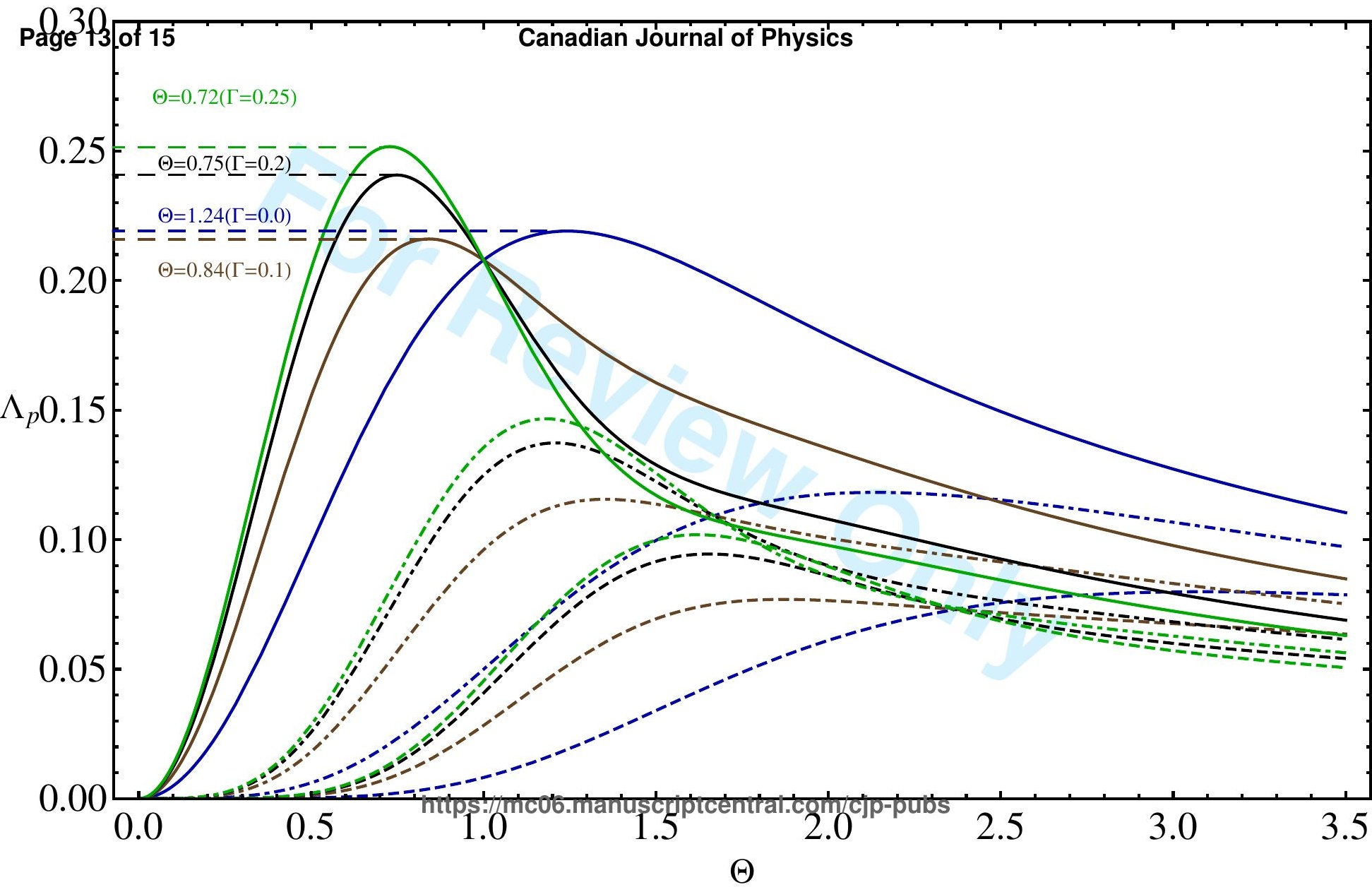




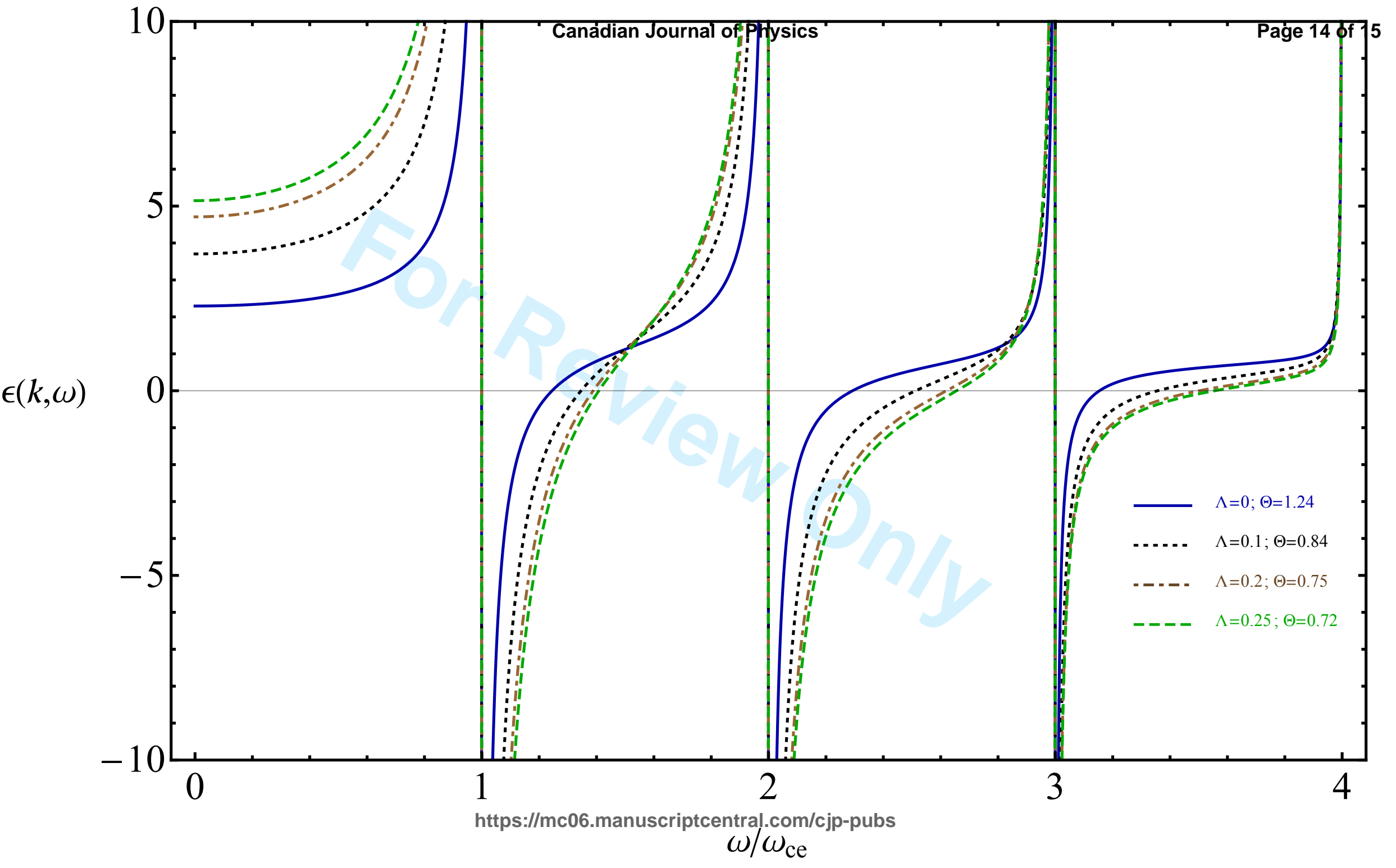




$$
\Gamma=0 \quad \Gamma=0.1 \quad \Gamma=0.2 \quad \Gamma=0.25
$$

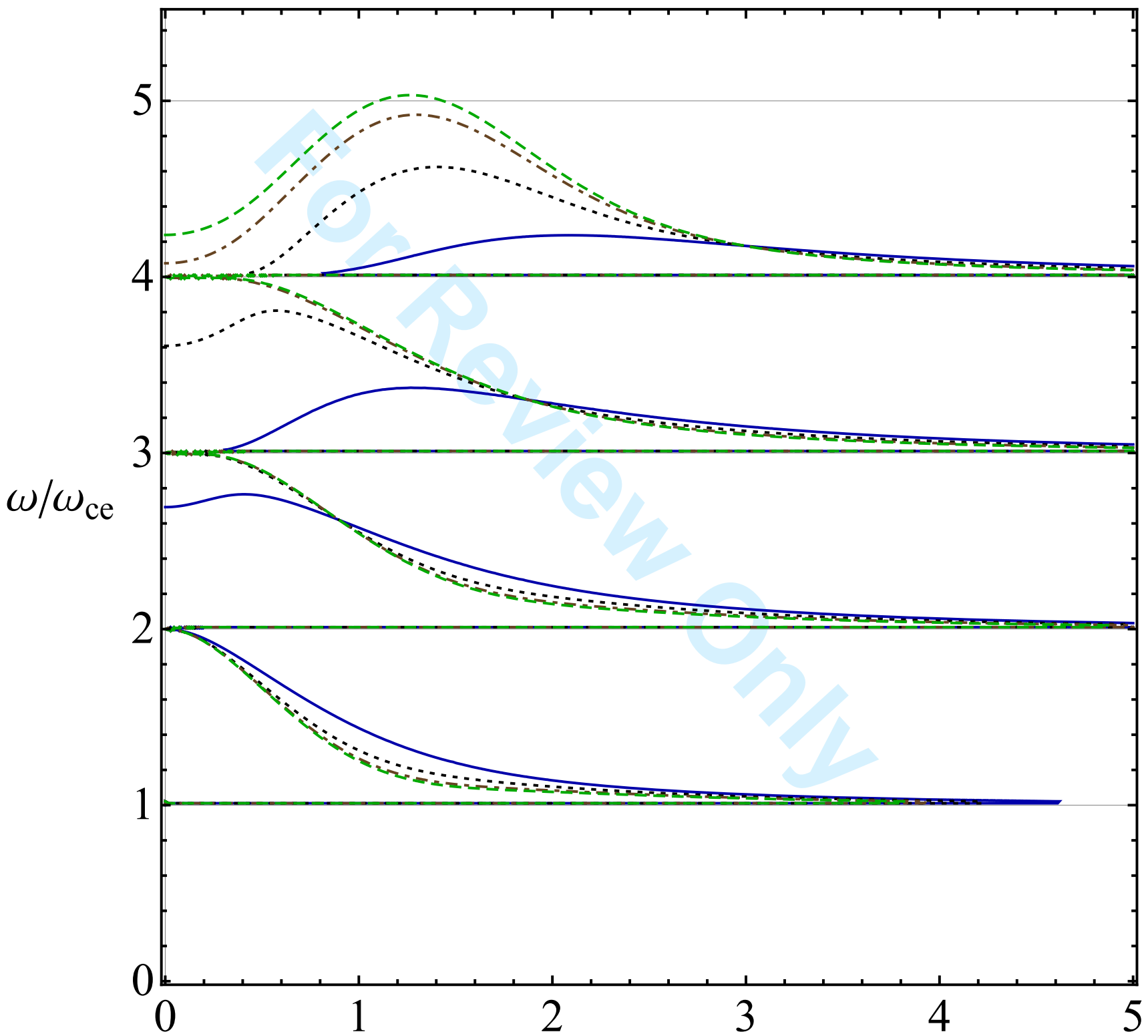

https://mc06.manuscriptcentral.com/cjp-pubs 